\title{
LA SITUACIÓN JURÍDICA DE LA EJECUCIÓN DE OBRAS POR ADMINISTRACIÓN DIRECTA
}

Yhon Cristian Barrionuevo Monzón *

\section{INFORMACIÓN DEL ARTÍCULO}

Art. Recibido: $23 / 06 / 2020$

Art. Aceptado: 04/08/2020

Art. Publicado: 10/08/2020 


\title{
RESUMEN:
}

El artículo se centra en demostrar que la ejecución de obras civiles por administración directa, no tiene un sustento legal en el derecho peruano, y que solo se sustenta en la costumbre y la falta de control, asimismo, se pretende demostrar que la Resolución de Contraloría Nro. 195-88-CG ha sido derogada tácitamente por el artículo 76 de la Constitución Política del Perú de 1993.

Palabras Claves: Ejecución de obras civiles, administración directa, Contrataciones con el Estado, Constitución Política de 1993 y derogación tácita.

\section{THE LEGAL STATUS OF THE EXECUTION OF WORKS BY DIRECT ADMINISTRATION}

\begin{abstract}
:
The article focuses on demonstrating that the execution of civil works by direct administration does not have a legal basis in Peruvian law, and is only based on custom and lack of control, specifically, it is supposed to demonstrate that the Comptroller's Resolution No. 195-88-CG has been tacitly repealed by article 76 of the 1993 Political Constitution of Peru.
\end{abstract}

Keywords: Execution of civil works, direct administration, Contracting with the State, Political Constitution of 1993 and tacit repeal. 


\section{INTRODUCCIÓN}

La contratación pública, nace de la obligación que tiene el estado de satisfacer el interés de la colectividad, siendo así, para llevar a cabo tal tarea y administrar correctamente los recursos, el estado puede proveerse de bienes, servicios y obras, los que por mandato constitucional son contratados y ejecutados obligatoriamente por proveedores privados; asimismo, cualquier excepción a esta regla es regulada por ley, conforme lo prevé el artículo 76 de la Constitución Política del Perú de 1993.

La contratación pública, nace de la obligación que tiene el estado de satisfacer el interés de la colectividad, siendo así, para llevar a cabo tal tarea y administrar correctamente los recursos, el estado puede proveerse de bienes, servicios y obras, los que por mandato constitucional son contratados y ejecutados obligatoriamente por proveedores privados; asimismo, cualquier excepción a esta regla es regulada por ley, conforme lo prevé el artículo 76 de la Constitución Política del Perú de 1993.

En una normalidad, lo expuesto en el párrafo precedente sería plenamente cumplido, sin embargo, existen ciertas anomalías en las obras públicas que nos motivan a llevar a cabo este artículo, nos centraremos básicamente en la ejecución de obras por administración directa, la cual es una modalidad anómala de ejecución de obras públicas en la que por costumbre las entidades del estado, con recursos propios (Obreros, ingenieros, etc.) se encargan de la ejecución de obras, las cuales crean diversas contingencias y problemas en su control, pues no existe una norma con rango de ley para la supervisión de estas.

Siendo así, esta modalidad de ejecución de obras públicas tiene base legal en la Resolución de Contraloría Nro. 185-88-CG, en la que se habilita a que la administración pública pueda ejecutar sus propias obras siempre que se pueda demostrar ciertas condiciones. Este tipo de modalidad de ejecución, no es acorde con el artículo 76 del texto constitucional, en la que se dispone que la ejecución de obras es obligatoriamente por contrata y licitación pública, asimismo, solo la ley dará las excepciones. En consecuencia, manifiestamente podemos apreciar que la Resolución de Contraloría Nro. 195-88-CG, no tiene rango de ley, por lo que no podría a primera vista regular una excepción al artículo 76 de la Constitución.

El artículo constará de 2 capítulos en los que desarrollaremos la contratación por administración directa y en una segunda parte las anomalías que convierten a esta modalidad de ejecución de obras en ilegal.

\section{LA CONTRATACIÓN POR ADMINISTRACIÓN DIRECTA}

En el Perú actualmente se pueden ejecutar obras civiles bajo dos modalidades: Por contrata y por administración directa. Siendo así, la ejecución de obras por administración directa, se puede definir según (PÉREZ, 2010) como: "esta modalidad supone que todos los elementos que componen la ejecución de la obra están a cargo de la Entidad, para ello es necesario que ésta posea la capacidad técnica y los elementos necesarios para tal efecto, debiendo contar también con toda la documentación necesaria; esta modalidad está regulada de modo alguno por lo dispuesto en la Resolución de Contraloría N 195-88-CG. En la práctica se refiere que es la modalidad por la que más se defrauda al fisco, cuando en la entidad no se tiene la voluntad sino de beneficiarse económicamente por la ejecución de la obra, además de que casi nunca 
Journal homepage: http://revistas.unap.edu.pe/rd/ E-mail: revistaderecho@unap.edu.pe

se cumplen los plazos establecidos en el cronograma de la obra, todo esto claro está, en perjuicio de la población beneficiaria."

La ejecución de obras por administración directa es regulada por la (Resolución de contraloría Nro. 185-88-CG, 1988) que consta de 2 artículos, en los que se dispone las condiciones para que una obra pueda ser ejecutada por Administración Directa, que son básicamente: Tener asignación presupuestal, equipos suficientes, tener el expediente técnico aprobado y demostrar que el costo de la ejecución debe ser menor o igual al que realizaría el privado con el presupuesto aprobado. Así lo ha especificado el artículo 1 de la Resolución de Contraloría antes descrita: "Artículo $1^{\circ}$. - APROBAR las siguientes normas que regulan la Ejecución de las Obras Públicas por Administración Directa. 1. Las Entidades que programen la ejecución de obras bajo esta modalidad, deben contar con: la asignación presupuestal correspondiente, el personal técnico-administrativo y los equipos necesarios. (...) 3. Es requisito indispensable para la ejecución de estas obras, contar con el "Expediente Técnico", aprobado por el nivel competente el mismo que comprenderá básicamente lo siguiente: memoria descriptiva, especificaciones técnicas, planos, metrados, presupuesto base con su análisis de costos y cronograma de adquisición de materiales y de ejecución de obra. En los casos que existan normas específicas referidas a la obra, se recabará el pronunciamiento del sector y/o entidad que corresponda. 4. La entidad debe demostrar que el costo total de la obra a ejecutarse por administración directa, resulta igual o menor al presupuesto base deducida la utilidad, situación que deberá reflejarse en la liquidación de la obra. (...)" por otro lado, el artículo 2 solo da disposiciones derogatorias. (Resolución de contraloría Nro. 195-88-CG, 1988)

Es importante resaltar que la (Resolución de contraloría Nro. 195-88-CG, 1988) es el único sustento en el que se ampara la ejecución de obras por administración directa, pues de una extensa revisión de la Ley de Contrataciones con el Estado, no encontramos artículo alguno que regule esta modalidad de ejecución. Afirmamos nuestra postura, indicando que (MUNICIPIOALDÍA, 2008) también llega a la misma conclusión: “¿Qué norma rige las obras por administración directa en lo referente a adicionales de obra y ampliaciones de plazo? No hay una norma específica que regule los adicionales de obra y ampliaciones de plazo en las obras por administración directa. La Resolución de Contraloría N¹95-88-CG regula las obras por administración directa, pero en ella no menciona nada respecto a adicionales de obra y ampliaciones de plazo. Esta norma (muy antigua, por cierto) tiene vacíos en muchos aspectos, pero está vigente hasta la fecha. Por otra parte, las normas del SNIP tampoco regulan específicamente el tema de adicionales de obra y ampliaciones de plazo en los proyectos, solo indica en el artículo $25^{\circ}$ sobre la verificación de viabilidad de los proyectos en ejecución, cuando hay modificaciones en el presupuesto, metas físicas, cronograma etc. siempre que no se modifique el objetivo del proyecto. Es decir, señala que se puede hacer tales modificaciones, pero no regula los términos detallados de los cambios a realizar."

Discrepamos de la página web, en el punto en el que se expone que esta norma es vigente, pues tenemos razones para entender la derogación tácita de la Resolución de Contraloría Nro. 195-88-CG, se dio ya en el año 1992. 
Journal homepage: http://revistas.unap.edu.pe/rd/ E-mail: revistaderecho@unap.edu.pe

\section{LAS ANOMALÍAS DE LA EJECUCIÓN DE OBRAS POR ADMINISTRACIÓN DIRECTA}

Teniendo una base clara de la ejecución de obras por administración directa, y siendo la base del artículo demostrar las anomalías que esta contiene, tenemos que desarrollar los antecedentes y el contexto en que esta norma fue emitida, teniendo en cuenta que esta resolución data del año 1988, es claro que el contexto y la base legal se ha modificado a la actualidad.

La Resolución de Contraloría Nro. 195-88-CG, fue emitida durante la vigencia de la (Constitución para la república del Perú de 1979,1980) teniendo amparo legal en el artículo 143 de la norma constitucional citada, la cual específicamente prevé: "Artículo 143. La contratación con fondos públicos de obras y suministros, así como la adquisición o enajenación de bienes se efectúan obligatoriamente por licitación pública. Hay concurso público para la contratación de servicios y proyectos cuya importancia y monto señala la ley de presupuesto. La ley establece el procedimiento, las excepciones y responsabilidades." (Constitución para la república del Perú de 1979, 1980), asimismo, el artículo 76 de la (Constitución Política del Perú de 1993, 1993) dispone: "Artículo 76.- Las obras y la adquisición de suministros con utilización de fondos o recursos públicos se ejecutan obligatoriamente por contrato y licitación pública, así como también la adquisición o la enajenación de bienes." (Constitución política del Perú de 1993, 1993)

En tal comparación, entendemos que el artículo 143 no prevé expresamente la obligación de contratar con un privado para el aprovisionamiento y ejecución de obras públicas, sin embargo, la figura del artículo 76 obliga necesariamente a la administración pública a que contrate con un privado a fin de que este ejecute la obra, concordamos con lo expresado por (Morón, 2017): "Sin embargo, si lo vemos con detenimiento apreciaremos que mientras la primera refiere que cuando se realicen contrato con fondos públicos, con los objetos que se señalan, estos deben concretarse previamente con la licitación pública como procedimiento de conformación, la segunda Carta afirma, por el contrario, que las actividades constructivas y suministros que las entidades se necesiten, se ejecutan mediante contratos (contrata), lo cual supone dos notas propias: alteridad (dos personas contrapuestas en la relación contractual) y patrimonialidad de la relación jurídica entre estas partes. Lo que la norma constitucionalidad no solo está planteado la necesidad de realizar licitaciones públicas para formar contratos, sino que cuando las entidades necesiten de obras, adquisición de bienes y adquisición de suministros con utilización de fondos o recursos públicos, estos se deben adquirir obligatoriamente por contrata."

Teniendo claro el contexto hasta este punto, entendemos que la ejecución de obras por administración directa, tenía plena armonía con la Constitución de 1979, debido a que en esta no se exigía la bilateralidad para la ejecución de obras públicas, sin embargo, las cuestiones y el contexto han variado hasta el momento, pues con el cambio de modelo económico, la Constitución de 1993, exige un roll pasivo del estado en cuestiones empresariales, esto se refleja en el artículo 76 de la mencionada carta magna, en la que se obliga a las entidades a que contraten con los privados a fin de ejecutar una obra pública.

En ese contexto, queda claro de que la Resolución de Contraloría Nro. 195-88-CG, es contradictoria al artículo 76 la Constitución de 1993, todo ello en vista del cambio 
Journal homepage: http://revistas.unap.edu.pe/rd/ E-mail: revistaderecho@unap.edu.pe

de contexto actual y el modelo económico del estado, por lo que se debería entender una derogación tácita de esta supuesta norma, pues es abiertamente contradictoria a la Constitución Política de 1993.

Ahora es preciso también aclarar en qué categoría jurídica recae esta Resolución, para así determinar si cumple con las condiciones que exige el artículo 76 para normar las excepciones a la contrata, pues, el último párrafo del artículo 76 de la Constitución, establece que la ley dará las excepciones, los procedimientos y las sanciones en materia de ejecución de la contratación pública. En todo caso lo que tenemos que hacer es determinar si la Resolución de Contraloría Nro. 195-88-CG, tiene el rango suficiente para considerarse una excepción a la norma constitucional.

En primer punto, analizamos qué es lo que la constitución quiere expresar, cuando menciona expresamente "la ley da las excepciones", para este punto, entendemos que solo las normas con rango de ley pueden ser las que limiten la ejecución de obras públicas por contrata, es decir, las Leyes del Congreso, los Decretos Supremos, los Decretos de Urgencia, y las normas que dan los organismos constitucionalmente autónomos: Municipios y Gobiernos Regionales. Sin embargo, por ser la Contratación Pública de interés nacional, solo podemos considerar que las limitantes al artículo 76 de la constitución pueden ser las leyes expresas del congreso y los Decretos Supremos que sean emitidos con facultades delegadas.

En ese sentido, en primer término, tenemos que analizar qué categoría tiene la Resolución de Contraloría Nro. 195-88-CG, esta resolución no tiene los presupuestos propios exigidos por la Constitución de 1993, pues no apreciamos que sea una Ley del Congreso y/o un Decreto Supremo emitido con facultades delegadas. Esto se sustenta también en la propia norma, que es suscrita por el titular de la Contraloría de 1988, en ese sentido, la Contraloría si bien es autónoma, no tiene las facultades para dictar normas de alcance nacional y que sean erga omnes.

Sin embargo, esta respuesta aún no satisface nuestras inquietudes, pues pretendemos ver en qué categoría de norma caería esta Resolución, para eso es menester inmiscuirnos en el origen de su emisión. La Resolución de Contraloría Nro. 195-88-CG, se sustenta en la ley nacional de control, aprobada mediante el (Decreto Ley Nro. 19039,1971) tal es así que esta norma se emitió en un contexto de estado de derecho, mas no en un estado constitucional de derecho. Por el estado de derecho entendemos que existía la supremacía de la ley, dejando en segundo plano a la Constitución, en ese sentido, en este Decreto Ley, emitido en el Gobierno Revolucionario de la Fuerza Armada, en el que Juan Velazco Alvarado era presidente, se manifestaba expresamente las facultades que tenía la contraloría para legislar en materia de contratación pública, vemos así los siguientes artículos contenidos en la parte considerativa de la Resolución de Contraloría Nro. 195-88-CG: "Estando a lo acordado en uso de las facultades contenidas en los incisos b) y k) del Artículo 12 e inciso e) del Artículo 16 de la Ley Orgánica del Sistema nacional de Control." (Resolución de Contraloría Nro. 195-88-CG, 1988)

Asimismo, estos artículos citados prevén expresamente lo siguiente: "Artículo 12.Son funciones de la Contraloría General de la República: (...) b. Dictar normas técnicas a seguirse en el proceso de control; (...) k. Dictar las disposiciones necesarias para asegurar el funcionamiento del proceso integral de control (...)" "Artículo 16.- 


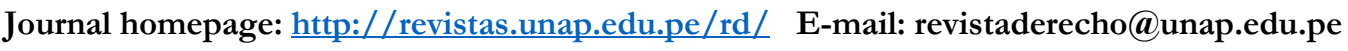

Corresponde al Contralor General de la República: (...) e. Dictar las normas técnicas de control las disposiciones que aseguren el funcionamiento de su proceso integral; (...)" (DECRETO LEY NRO. 19039,1971)

En ese contexto, podríamos decir que para el año 1988, la Resolución de Contraloría Nro. 195-88-CG, tenía plena vigencia legal y hasta sería considerada una norma con rango de ley.

Continuando con la breve reseña histórica, la base de la Resolución de Contraloría Nro. 195-88-CG, que es el Decreto Ley Nro. 19039, que otorga facultades expresas de normar al contralor, ha sido derogada por la (Ley Nro. 26162, 1992) que entra en vigencia el 30 de diciembre de 1992 (deperu.com, s.f.) en ese sentido, la base legal de la norma que regula la ejecución de obras por administración directa ha sido derogada, lo que supondría la derogación tácita de la Resolución de Contraloría Nro. 195-88-CG. A la vez, la Ley Orgánica del Sistema Nacional de Control y de la Contraloría General de la República (Ley Nro. 27785), en la segunda disposición final, ha derogado la (Ley Nro. 26162. 2002).

Respecto a la derogación, el artículo 103 de la Constitución Política de 1993, regula: “(...) una ley solo se deroga por otra ley”; asimismo, el artículo I del Título Preliminar del Código Civil regula: "La ley se deroga sólo por otra ley. La derogación se produce por declaración expresa, por incompatibilidad entre la nueva ley y la anterior o cuando la materia de ésta es íntegramente regulada por aquella. Por la derogación de una ley no recobran vigencia las que ella hubiere derogado." (Decreto Legislativo Nro. 295, 1984).

En ese sentido, las facultades delegadas al contralor, contenidas en el Decreto Ley Nro. 19039, en mérito a las que ha sido emitida la Resolución de Contraloría Nro. 19588-CG ha sido derogada, por lo que la Resolución que regula las obras por administración directa, ya no tendría un sustento en el ordenamiento jurídico, de la misma manera, un sentido más amplio para entender que la norma ha sido derogada, es la clara incompatibilidad que existe con la Constitución de 1993, la contradicción a la constitución fue resuelta por el tribunal constitucional, en ese sentido en el EXP. N. ${ }^{\circ}$ 00025-2010-PI/TC, este tribunal expresó en el fundamento 16: “16. (ii) Una situación distinta es la que ocurre en aquellos casos en los que los conflictos de normas se presentan entre ley preconstitucional y Constitución nueva, ya que, además de resolverse con base en el principio lex superior derogat lex inferiorem, la antinomia también puede solucionarse bajo la aplicación del criterio lex posteriori derogat lex priori. En tal hipótesis, además de norma superior, la Constitución nueva también es una norma posterior, de modo que la inaplicación de la ley, como consecuencia de su derogación tácita, puede realizarla cualquier órgano estatal. Eso fue lo que se expresó en la STC 0010-2001-AI/TC [que luego se ha reiterado en la STC 0017-2003-AI/TC], en la que después de afirmar también la competencia del Tribunal para controlar la validez constitucional de las leyes anteriores a la Constitución de 1993, se destacó que ello era sin perjuicio de reconocer "que eventualmente esa misma legislación pueda considerarse derogada tácitamente, en aplicación del principio lex posterior derogat priori. Este último criterio, que sirve para resolver una antinomia entre dos normas en el tiempo, es una manifestación de los efectos derogatorios que tiene una Constitución, que es una auténtica norma jurídica suprema [Cf. fundamento 24], precisando, en la STC 0011-2004-AA/TC, que 
Journal homepage: http://revistas.unap.edu.pe/rd/ E-mail: revistaderecho@unap.edu.pe

"la observación de que una ley pre-constitucional ha(ya) quedado derogada tácitamente por la entrada en vigencia de una Constitución nueva es un asunto que incluso puede efectuarse en sede administrativa, (a diferencia de) la inaplicación de una ley por ser contraria a la Constitución (que) es una competencia que sólo pueden ejercer los jueces (artículo 138 ${ }^{\circ}$ ) [fundamento 4]." (Demanda de inconstitucionalidad interpuesta por el Presidente de la República contra la ordenanza regional $\mathrm{N}^{\circ} 004-$ 2010-GRA/CR, emitida por el gobierno regional de Ayacucho, 2010).

He aquí nuestra preocupación, pues en el Perú hasta el año 2018, la cantidad de obras públicas iniciadas y reportadas sumaron: 9,072, de las cuales la mayoría son aquellas ejecutadas por contrata (5.773) y administración directa $(3,190)$, constituyendo las obras bajo administración directa un 35\% (Gestión, 2019), asimismo, actualmente en la ciudad de Puno, se viene ejecutando el Estadio Nacional de la UNA-Puno por administración directa, la cual tiene un valor inicial de 45 millones de soles.

Actualmente, las obras públicas siguen siendo ejecutadas por administración directa, teniendo en cuenta la derogación tácita de la Resolución de Contraloría Nro. 195-88-CG, y además el factor de que la propia administración de oficio puede observar que una norma ha sido derogada por la Constitución Política del Perú de 1993. En consecuencia, entendemos que la contratación por administración directa, no tendría una base legal que la respalde y solo es guiada por la falta de sanción del ente fiscalizador y la aceptación de la costumbre de las entidades del estado por ejecutar sus obras.

Hasta este punto hemos demostramos que existen 2 imprecisiones sumamente graves para la ejecución de obras por administración directa, lo que nos conlleva a afirmar que esta contratación es contraria a la Constitución, además de que no tendría base legal para ser usada, en el mismo modo, si se intenta justificar esta modalidad en la costumbre (Que es el único sustento), tendríamos un problema más grande aún, pues la costumbre no es fuente del derecho administrativo, por lo que no puede normalizar una práctica jurídica que es abiertamente contraria a la Constitución.

Suma a nuestras preocupaciones, la categoría jurídica en la que recaerían las obras públicas ya ejecutadas por administración directa, pues existen algunas que ya fueron liquidadas y están siendo usadas por la población, pensamos que solo debería persistir la responsabilidad de quien permitió la ejecución de las obras por esta modalidad, sin embargo, este es tema de otro artículo.

Finalmente, indicamos que según (Gestión, 2018), la contraloría ha presentado un proyecto de Decreto de Urgencia a fin de normar la obra por administración directa, a fin de coadyuvar procesos de selección en la que las convocatorias han sido declaradas desiertas, pensamos que la contraloría, es consciente de las anomalías que hemos expuesto en nuestra investigación, por lo que pretende regular con una norma con rango de ley las excepciones a la ejecución de obras por contrata y regular adecuadamente la ejecución de obras por administración directa, por lo que creemos que esta modalidad de ejecución contractual será prontamente regulada y adaptada al régimen económico y a las exigencias de la época actual. 


\section{CONCLUSIÓN}

La modalidad de ejecución de obras civiles por administración directa, no tiene sustento legal en el ordenamiento jurídico peruano, pues el asidero legal de esta, Resolución de Contraloría Nro. 195-88-CG, es claramente contradictoria al artículo 76 de la Constitución Política del Perú de 1993.

En esa línea de ideas, de acuerdo al criterio lex superior derogat lex inferiorem y lex posteriori derogat lex priori, expuestos por el Tribunal Constitucional en el expediente Nro. 00025-2010-PI/TC, podemos encontrar que existe un criterio suficiente para que los órganos estatales puedan inaplicar la Resolución de Contraloría Nro. 195-88-CG, atendiendo a la expresa contradicción de esta con la Constitución; y por si esto no fuera suficiente, en el supuesto negado de que esta norma siga vigente, no cumpliría con el rango legal suficiente para imponer una excepción al artículo 76 de la Constitución Política del Perú, en vista de que no es una norma con rango de Ley.

Finalmente, a modo de recomendación y atendiendo a la propuesta hecha por la Contraloría General de la República por regular esta modalidad de contratación, es menester pronunciarnos respecto a si esta modalidad de ejecución de obras civiles debería ser permitida; a nuestro parecer la respuesta es no, pues ninguna práctica (así se sustente en la eficiencia) puede justificar el quebrantamiento de la Constitución, más aún si esta modalidad de ejecución de obras, es la que más problemas trae al estado $\mathrm{y}$ al fisco. Adicionalmente, podríamos estar de acuerdo con una regulación armoniosa con el artículo 76 de la Constitución, que respete en sentido estricto el carácter de excepcionalidad de esta modalidad, dando la oportunidad en primer lugar al privado y en caso de que no hubiere un privado dispuesto a ejecutar, permitir a la administración pública a que ejecute sus propias obras. 
Journal homepage: http://revistas.unap.edu.pe/rd/ E-mail: revistaderecho@unap.edu.pe

\section{REFERENCIAS}

CÓDIGO CIVIL, Decreto Legislativo Nro. 295. Diario Oficial el peruano, Lima, Perú, 25 de julio de 1984

CONSTITUCIÓN POLÍTICA DEL PERÚ DE 1993. Diario Oficial el peruano, Lima, Perú, 29 de diciembre de 1993

CONSTITUCIÓN PARA LA REPÚBLICA DEL PERÚ DE 1979. Diario Oficial el peruano, Lima, Perú, 28 de julio de 1980

DEMANDA DE INCONSTITUCIONALIDAD interpuesta por el Presidente de la República contra la Ordenanza Regional Nº04-2010-GRA/CR, emitida por el Gobierno Regional de Ayacucho, Expediente Nro. 00025-2010-PI/TC (Tribunal Constitucional, 2010)

DEPERU (s.f.) Deperu.com. Lima, Perù; rescatado de https://www.deperu.com/legislacion/derogada-decreto-ley-num-19039.html

GESTIÓN. (30 de noviembre de 2019). Contraloría propone regular ejecución de obras públicas por administración directa. Recuperado de https://gestion.pe/economia/contraloria-propone-regular-ejecucion-deobras-publicas-por-administracion-directa-nndc-noticia/?ref=gesr

LEY DEL SISTEMA NACIONAL DE CONTROL, Ley Nro. 26162, Diario Oficial el peruano, Lima, Perú, 29 de diciembre de 1992

LEY ORGÁNICA DEL SISTEMA NACIONAL DE CONTROL DE LA ACTIVIDAD PÚBLICA, Decreto Ley Nro. 19039. Diario Oficial el peruano, Lima, Perú, 16 de noviembre de1971

LEY ORGÁNICA DEL SISTEMA NACIONAL DE CONTROL Y DE LA CONTRALORÍA GENERAL DE LA REPÚBLICA, Ley Nro. 26162. Diario Oficial el peruano, Lima, Perú, 23 de julio de 2002

MORÓN URBINA, J. C. (2016). Las Obras por Administración Directa y por encargo: ¿Solución o problema? ¿Hay que restringirlas?. IUS ET VERITAS, 24(53), 246260. Recuperado a partir de http://revistas.pucp.edu.pe/index.php/iusetveritas/article/view/16546

MUNICIPIOALDÍA. (noviembre de 2008). ¿Qué norma rige las obras por administración directa en lo referente a adicionales de obra y ampliaciones de plazo? Recuperado de https://municipioaldia.com/consultas-delmes/adicionales de obra y ampliacion de plazo/

PÉREZ DEZA, P. (8 de diciembre de 2010). Patrickeperezdeza [Blog]. Recuperado de http://blog.pucp.edu.pe/blog/patrickeperezdeza/tag/resolucion-de-contraloria-n$195-88-\mathrm{cg} /$

RESOLUCIÓN DE CONTRALORÍA NRO. 195-88-CG, Diario Oficial el peruano, Lima, Perú, 18 de julio de 1988 